\title{
Infrared Studies of the Onset of Conductivity in Ultra-Thin Pb Films
}

\author{
P.F. Henning ${ }^{1,2}$, C.C. Homes ${ }^{1}$, S. Maslov ${ }^{1}$, G.L. Carr ${ }^{1}$, D.N. Basov ${ }^{2}$, B. Nikolić ${ }^{3}$, and M. Strongin ${ }^{1}$ \\ ${ }^{1}$ Department of Physics, Brookhaven National Laboratory, Upton, NY 11973 \\ ${ }^{2}$ Department of Physics, University of California at San Diego, La Jolla, CA 92093 \\ ${ }^{3}$ Department of Physics, SUNY at Stony Brook, Stony Brook, NY 11794
}

(August 19, 2019)

\begin{abstract}
In this paper we report the first experimental measurement of the infrared conductivity of ultrathin quenched-condensed $\mathrm{Pb}$ films. For dc sheet resistances such that $\omega \tau \ll 1$ the ac conductance increases with frequency but is in disagreement with the predictions of weak localization. We attribute this behavior to the effects of an inhomogeneous granular structure of these films, which is manifested at the very small probing scale of infrared measurements. Our data are consistent with predictions of two-dimensional percolation theory.
\end{abstract}

PACS numbers: 78.66.-w, 74.76.Db, 72.15.Rn, 64.60.Ak

Transport measurements in ultra-thin films have been a subject of active interest over many years [1]. These systems, consisting of a thin layer of metal deposited onto a substrate held at LHe temperatures, provide a relatively simple way to study the interplay between localization, electron-electron interactions, and superconductivity in disordered quasi-2D metals. These experiments are in quantitative agreement with predictions of localization theory [2] combined with the effects of diffusionenhanced electron-electron interactions [3]. The reason why these theories, developed for homogeneous materials, work so well in the case of granular, inhomogeneous films is that the length scale at which electrons lose phase coherence in these measurements is usually much larger than the characteristic size of inhomogeneities (grains, percolation clusters, etc.) of the film.

Yet another way to modify the length scale is by simply changing the probing frequency. This gives rise to frequency dependence of the ac conductivity in the region $\omega \tau \ll 1$, where the Drude theory predicts a plateau. While the experimental data on the frequency dependence of conductivity are virtually non-existent for ultrathin quenched-condensed films, they abound for thicker, more granular films, deposited onto a warm substrate [ 4 ]. In ac conductivity the frequency itself defines a characteristic dephasing length scale $L_{\omega}=\sqrt{D / \omega}$, where $D$ is a diffusion coefficient. In the frequency range where $L_{\omega}$ is smaller than other dephasing length scales, it enters into all localization and interaction formulas and gives rise to frequency-dependent quantum corrections to the conductivity. However, these quantum effects constitute only one source of frequency dependence of the conductivity. In the region where the material is strongly inhomogeneous on the scale of $L_{\omega}$, the frequency dependence of conductivity is dominated by purely classical effects due to charge dynamics on a network of capacitively and resistively-coupled clusters of grains. The effective way to describe these effects theoretically is provided by the framework of percolation theory [5]. In this theoreti- cal approach the ac conductivity is shown to increase with frequency. Indeed, since capacitive coupling between grains is proportional to frequency, grains become more and more connected as the frequency is increased. It is also known that purely quantum effects such as localization and diffusion-enhanced interaction corrections become profoundly modified on length scales where the material can no longer be treated as homogeneous [6]. The presence of inhomogeneities is known to change the functional dependence of these corrections on the phase coherence length, while leaving the absolute magnitude of the effect virtually unchanged.

In this letter we report the first measurement of conductivity at infrared frequencies in ultra-thin films. Films used in this experiment were made in situ by evaporating $\mathrm{Pb}$ onto a $\mathrm{Si}(111)$ (sets 1 and 2) and glass (set 3) substrates, mounted in an optical cryostat, held at $10 \mathrm{~K}$. Ag tabs, pre-deposited onto the substrate, were used to monitor the dc resistance of the film. Infrared transmission measurements from 500 to $5000 \mathrm{~cm}^{-1}$ (set 1), and 2000 to $8000 \mathrm{~cm}^{-1}$ (sets 2 and 3 ) were made using a Bruker $113 \mathrm{v}$ spectrometer at the new high-brightness U12IR beamline at the BNL's National Synchrotron Light Source. The substrates were covered with a $5 \AA$ thick layer of Ge to promote two-dimensional thin-film growth, rather than the agglomeration of the deposited $\mathrm{Pb}$ in larger grains. Even when using this traditional method, which is known to promote the growth of more homogeneous films, where the continuity has been measured at near a monolayer of deposited metal, we only saw the beginnings of continuity near $8-20 \AA$ of metal with consistently thinner films using the glass substrates. Films were evaporated at pressures ranging from the low $10^{-8}$ to the mid $10^{-9}$ Torr range. The transmission spectra were obtained after successive in-situ $\mathrm{Pb}$ depositions. The dc resistances in set 1 on $\mathrm{Si}$ range from $64 \mathrm{M} \Omega / \square$ at $17.4 \AA$ average thickness to $543 \Omega / \square$ at $70 \AA$. The $70 \AA$ sample was then annealed twice, first to $80 \mathrm{~K}$, and then to $300 \mathrm{~K}$. As a result its resistance at $10 \mathrm{~K}$ became $166 \Omega / \square$ after the 
first annealing, and $100 \Omega / \square$ after the second annealing. Films from set 2 (also on $\mathrm{Si}$ ) are similar to set 1: we have observed $R_{\square}=20 \mathrm{M} \Omega / \square$ at $18 \AA$ and $R_{\square}=1000 \Omega / \square$ at $88 \AA$. Finally, films from set 3 , deposited on a Gecoated glass substrate, range from 13 to $200 \AA$, while $R_{\square}$ changes between $5.6 \mathrm{M} \Omega$ and $22.8 \Omega$.

The transmission coefficient of a film deposited on the substrate, measured relative to the transmission of the substrate itself, is related to real and imaginary parts of the sheet conductance of the film as [7]

$$
\mathrm{T}(\omega)=\frac{1}{\left[1+Z_{0} \sigma_{\square}^{\prime}(\omega) /(n+1)\right]^{2}+\left(Z_{0} \sigma_{\square}^{\prime \prime}(\omega) /(n+1)\right)^{2}} .
$$

Here $Z_{0}=377 \Omega$ is the impedance of free space, $n$ is the index of refraction of the substrate, equal to $n_{S i}=3.315$ for silicon and $n_{G}=1.44$ for glass, and $\sigma_{\square}^{\prime}(\omega)$ (sometimes called $G), \sigma_{\square}^{\prime \prime}(\omega)$ are the real and imaginary parts of the sheet conductance of the film. Almost everywhere in our experiments $\sigma_{\square}^{\prime}(\omega), \sigma_{\square}^{\prime \prime}(\omega) \ll(n+1) / Z_{0}$. In this case the contribution of the imaginary part of conductance to the transmission coefficient is negligible and Eq. (1) can be approximately replaced by $\mathrm{T}(\omega) \simeq\left[1+\mathrm{Z}_{0} \sigma_{\square}^{\prime}(\omega) /(\mathrm{n}+1)\right]^{-2}$. Even for our thickest films, where $\sigma_{\square}^{\prime \prime}(\omega) \approx(n+1) / Z_{0}$, the error in calculating $\sigma_{\square}^{\prime}(\omega)$ in this way is less than $10 \%$ over our frequency range. Throughout the manuscript we will use this approximation to extract the real part of the sheet conductance of the film from its transmission coefficient. Only for our thickest films will we use Eq. (1) to derive parameters of Drude fits.

In Fig. 1 we plot the frequency-dependent conductance, extracted from the transmission data for the films from the set 3 with the help of the above approximation to Eq. (11). The seven thickest films from this set exhibit a characteristic Drude falloff at high frequencies. For the rest of the films the conductivity systematically increases with frequency throughout our frequency range. The inset in Fig. 1 shows the average ac conductance as well as the dc sheet conductance for set 3 as a function of thickness. Note the curves start to significantly deviate from each other at around $50 \AA$.

In order to fit the conductance of our thickest films with the Drude formula, one needs to use the untruncated Eq. (11) for the transmission coefficient. Inserting the Drude expression for the sheet conductance $\sigma_{\square}(\omega)=\sigma_{D} /(1-i \omega \tau)$ directly into Eq. 11 one gets $\mathrm{T}(\omega) /[1-\mathrm{T}(\omega)]=\left(1+\omega^{2} \tau^{2}\right) /\left[\left(\sigma_{D} / \sigma_{0}\right)^{2}+2 \sigma_{D} / \sigma_{0}\right]$, where $\sigma_{0}=(n+1) / Z_{0}$. Therefore, the transmission data, which are consistent with the Drude formula can be fitted with a straight line, when $\mathrm{T}(\omega) /[1-\mathrm{T}(\omega)]$ is plotted as a function of $\omega^{2}$. In Fig. 2 we plot the 7 thickest films from the set 3 in this way. The knowledge of the average thickness of our films along with parameters of the Drude formula enables us to calculate the plasma frequencies in our films. They are shown in the inset of Fig. 2 as a function of $1 / \sigma_{D}$ - the dc sheet resistance in Drude formula, which itself was extracted from our Drude fit. These results are in excellent agreement with the experimentally determined lead plasma frequency of $\omega_{p}=59400 \mathrm{~cm}^{-1}$ [8]. In the remainder of the manuscript we discuss possible interpretations of the increase of the conductance with frequency, which we observe in our thinner films.

One mechanism which is known to cause a frequency dependence of conductivity within a Drude plateau $(\omega \tau \ll 1)$ is purely quantum mechanical in origin. The conductivity is known to be reduced due to increased back scattering of phase-coherent electrons (so called weak localization (WL) [2]), as well as diffusion enhanced electron-electron interactions (EEI) [3]. The magnitude of this reduction depends on the length scale over which an electron maintains its phase coherence. In the absence of external magnetic field or ac electric field this length is determined by the temperature. It is given by the inelastic scattering length $l_{i n}(T)=\sqrt{D \tau_{\text {in }}(T)}$ for WL, and the thermal coherence length $L_{T}=\sqrt{\hbar D / k T}$ for EEI. Here $D$ is the diffusion coefficient of the electron related to its dc conductivity by the Einstein formula $\sigma=e^{2}(d N / d \mu)_{E_{F}} D$, and $\tau_{i n}(T)$ is the temperature dependent inelastic scattering (dephasing) time. In the presence of the ac electric field the diffusive motion of an electron is restricted to a spatial region of size $L_{\omega}=\sqrt{D / \omega}$. If this length scale turns out to be shorter than the corresponding dc length scale, it is $L_{\omega}$ which enters in all WL and EEI formulas.

The question of effective dimensionality of the quasi$2 \mathrm{D}$ sample is decided by comparing $L_{\omega}$ to the film thickness $d$. The frequency dependent WL corrections to the sheet conductance of the film are given by $\Delta \sigma_{\square}^{2 D}(\omega)=$ $\frac{e^{2}}{2 \pi^{2} \hbar} \ln \omega \tau$ in the 2D limit $\left(d<L_{\omega}\right)$ [2], and $\Delta \sigma_{\square}^{3 D}(\omega)=$ $\frac{\sqrt{2} e^{2}}{4 \pi^{2} \hbar} d \sqrt{\frac{\omega}{D}}$ in the 3D limit $\left(d>L_{\omega}\right)[9]$. At the lower end of our frequency range $\omega=500 \mathrm{~cm}^{-1}$ for a realistic value of $D=5 \mathrm{~cm}^{2} / \mathrm{s}$ we can estimate $L_{\omega} \leq 20 \AA \leq d$. Therefore, for our films one should use the formulas of three-dimensional localization theory. The frequencydependent sheet conductance in most of our films is consistent with the $\sqrt{\omega}$ dependence of 3D WL. However, we believe that in order to explain the frequency dependence of our experimental data one needs to look for yet another mechanism, supplementing that due to weak localization and electron-electron interactions. The problems with ascribing the observed frequency dependence of conductivity solely to WL and EEI effects are: (i) the dependence of the slope of the conductivity vs $\sqrt{\omega}$ on the thickness of the film and the dc sheet conductance, which determines the diffusion coefficient $D$, does not agree with predictions of the $3 \mathrm{D}$ localization. (ii) the weak localization theory is only supposed to work in the limit where its corrections are much smaller than the dc conductivity. In our experimental data we don't see any change of be- 
havior as the corrections to conductivity become bigger than the dc conductivity. In fact the $\sqrt{\omega}$ fit works very well and gives roughly the same slope even for films with dc sheet resistance of $\approx 100 \mathrm{k} \Omega$, while the ac sheet resistance is only $\approx 1 \mathrm{k} \Omega$. Furthermore, the $3 \mathrm{D}$-localization theory predicts that the $\sqrt{\omega}$ dependence of weak localization theory should be replaced by $\omega^{(d-1) / d}=\omega^{1 / 3}$ dependence at or near the 3D metal-insulator transition [10]. In our experimental data we see no evidence for such a crossover.

There exists yet another, purely classical effect that gives rise to the frequency dependence of the conductivity. It is relevant in strongly inhomogeneous, granular films. There is ample experimental evidence that even ultra-thin quenched-condensed films have a microscopic granular structure 11, 12]. In order to describe the ac response of a film with such a granular microstructure one needs to know the geometry and conductivity of individual grains as well as the resistive and capacitive couplings between grains. The disorder, which is inevitably present in the placement of individual grains, makes this problem even more complicated. However, there exist two very successful approaches to the analytical treatment of such systems. One of them, known as the effective-medium theory [13], can be viewed as a meanfield version of a more refined approach, based on scaling near the percolation transition. The EMT takes into account only concentrations of metallic grains and the voids between the grains, disregarding any spatial correlations. A more refined approach takes into account the geometrical properties of the mixture of metallic grains and voids. The insulator-to-metal transition in this approach is nothing else but the percolation transition, in which metallic grains first form a macroscopic connected path at a certain critical average thickness $d_{c}$ of the film. The dc conductivity above the transition point scales as $\left(d-d_{c}\right)^{t}$, where $t=1.3$ in $2 \mathrm{D}$ and $t=1.9$ in 3D [5]. Just below the percolation transition the dielectric constant of the medium diverges as $\epsilon(d) \sim\left(d_{c}-d\right)^{-s}$, where $s=1.3$ in $2 \mathrm{D}$ and $s=0.7$ in $3 \mathrm{D}$. The diverging dielectric constant is manifested as the imaginary part of the ac conductivity $\sigma(\omega) \sim-i \omega\left(f_{c}-f\right)^{-s}$. In general the complex ac conductivity of the metal-dielectric (void) mixture close to the percolation transition is known [5] to have the following scaling form:

$$
\sigma(\omega, d)=\left|d-d_{c}\right|^{t} F_{ \pm}\left(-i \omega\left|d-d_{c}\right|^{-(t+s)}\right) .
$$

Here $F_{+}(x)$ and $F_{-}(x)$ are scaling functions above and below the transition point correspondingly. Note that this scaling form correctly reproduces the scaling of the dc conductivity above the transition and the divergence of the dielectric constant below the transition provided that $F_{+}(x)=F_{+}^{(0)}+F_{+}^{(1)} x+F_{+}^{(2)} x^{2}+\ldots$, while $F_{-}(x)=F_{-}^{(1)} x+F_{-}^{(2)} x^{2}+\ldots$ One should mention that the predictions of the EMT can also be writ- ten in this scaling form with mean-field values of the exponents $t=s=1$, and scaling functions $F \pm(x)=$ $\left(\sqrt{D^{2}+4(D-1) x} \pm D\right) /(2(D-1))$, where $D$ is the spatial dimension.

Since the metallic grains in our films form not more than two layers, our data should be interpreted in terms of the two-dimensional percolation theory. In two dimensions $t=s=1.3$ [5], and according to Eq. (2) the ac conductivity precisely at the transition point $d=d_{c}$ is given by $\sigma\left(\omega, d_{c}\right)=A\left(i \omega / \omega_{0}\right)^{t /(t+s)}=A\left(i \omega / \omega_{0}\right)^{1 / 2}$. This prediction is in agreement with our experimental data. In Fig. 3 we attempt the rescaling of our data according to Eq. (2). The critical thickness $d_{c}$ is determined as the point where the ac conductivity divided by $\sqrt{\omega}$ is frequency independent. Of course, the experimental uncertainty in our data points does not allow us to determine which exponents $t$ and $s$ provide the best data collapse. However, as we can see from Fig. 3 our data are consistent with the scaling form of the 2D percolation theory. Finally, we use Fig. 3 to estimate basic parameters such as typical resistance $R$ of an individual grain and typical capacitance $C$ between nearest neighboring grains. From the limiting value of $\sigma(\omega, d)\left(d_{c} /\left|d-d_{c}\right|\right)^{1.3}$ at small values of the scaling variable $x=\omega\left(d_{c} /\left|d-d_{c}\right|\right)^{2.6}$ for $d>d_{c}$, one estimates the conductance of an individual grain to be of order of $R \sim 1000 \Omega$. In the simplest $R C$ model, where the fraction of the bonds of the square lattice are occupied by resistors of resistance $R$, while the rest of the bonds are capacitors with capacitance $C$, the ac conductivity exactly at the percolation threshold is given by $A / R(i \omega R C)^{1 / 2}$, where $A$ is a constant of order of one. Therefore, the slope $\partial \sigma / \partial \sqrt{\omega}$ in our system should be of the same order of magnitude as $\sqrt{R C} / R$. This gives $C \simeq 2.6 \times 10^{-19} \mathrm{~F}$, which is in agreement with a very rough estimate of the capacitance between two islands $200 \AA \times 200 \AA \times 30 \AA$ separated by a vacuum gap of some $20 \AA$, giving $C \simeq 2.7 \times 10^{-19} \mathrm{~F}$. This order of magnitude estimate confirms the importance of taking into account inter-island capacitive coupling when one interprets the ac conductivity measured in our experiment. Indeed, $R=1000 \Omega$, and $C=3 \times 10^{-19} \mathrm{~F}$ define a characteristic frequency $1 / R C \simeq 17000 \mathrm{~cm}^{-1}$ comparable to our frequency range.

In summary, we have measured the conductivity of ultra-thin $\mathrm{Pb}$ films in the frequency range 500 to $8000 \mathrm{~cm}^{-1}$. The evolution of $\sigma(\omega)$ with DC sheet resistance is consistent with classical two-dimensional percolation theory in this range. At lower probing frequencies, where $L_{\omega}$ becomes larger than the scale of inhomogeneities in these films, we expect that the effects of weak localization will become more prevalent.

We have benefited from fruitful discussions with P.B. Allen, A.M. Goldman, V.J. Emery, V.N. Muthukumar, Y. Imry, Z. Ovadyahu, and M. Pollak. The work at Brookhaven was supported by the U.S. Department of Energy, Division of Materials Sciences, under contract 
no. DE-AC02-98CH10886. Support from NSF grants DMR-9875980 (D.N.B.) and DMR-9725037 (B.N.) is acknowledged. Research undertaken at NSLS is supported by the U.S. DOE, Divisions of Materials and Chemical Sciences.
FIG. 3. The rescaling of conductivity data in set 3 according to predictions of the percolation theory. The value for $d_{c}$ is $34 \AA$.

[1] See, for example, M. Strongin, R.S. Thompson, O.F. Kammerer and J.E. Crow, Phys. Rev. B 1, 1078 (1970); E.G. Astrakharchik and A.I. Shalnikov, Sov. Phys. JETP 45, 844 (1977); J.M. Graybeal and M.R. Beasley, Phys. Rev. B 29, 4167 (1984); R.C. Dynes, A.E. White, J.M. Graybeal, and J.P. Garno, Phys. Rev. Lett. 57, 2195 (1986); N. Markovic, C. Christiansen, and A. M. Goldman, Phys. Rev. Lett. 81, 5217 (1998); S.-Y. Hsu and J.M. Valles, Jr., Phys. Rev. Lett. 74, 2331 (1995).

[2] For a review see P.A. Lee and T.V. Ramakrishnan, Rev. Mod. Phys. 57, 287 (1985).

[3] For a complete discussion and many early references, see also B.L. Altshuler and A.G. Aronov, in ElectronElectron Interaction in Disordered Systems, edited by A.L. Efros and M. Pollak (North Holland, 1985).

[4] D.B. Tanner, Y.H. Kim, and G.L. Carr, Mat. Res. Soc. Symp. Proc. 195, 3 (1990).

[5] An exhaustive review can be found at J.P. Clerc et. al., Advances in Physics 39, 191 (1990).

[6] Y. Gefen, D.J. Thouless, and Y. Imry, Phys. Rev. B 28, 6677 (1983).

[7] M. Tinkham, "Introduction to Superconductivity", McGraw-Hill, New York, p. 70, 1975.

[8] M.A. Ordal et. al, Applied Optics 24, 4493 (1985).

[9] L.P. Gorkov, A.I. Larkin, and D.E. Khmelnitskii, JETP Lett. 30, 248 (1979).

[10] D. Vollhardt and P. Wölfle, Phys. Rev. Lett. 48, 699 (1982).

[11] K.L. Ekinci and J.M. Valles, Jr., Phys. Rev. B 58, 7347 (1998) and Phys. Rev. Lett. 82, 1518 (1999).

[12] K.Kagawa, et al, Phys. Rev. B 53, R2979 (1996).

[13] D.A.G. Bruggeman Ann. Phys. (Leipzig) 24, 636 (1935).

FIG. 1. Sheet conductance vs frequency for set 3 . The dashed lines plotted between 3000 and $4000 \mathrm{~cm}^{-1}$ (where the glass substrate is opaque) are a guide to the eye. The inset shows the inverse average conductance in our frequency range (filled symbols) and the dc sheet resistance (open symbols) as a function of the film thickness.

FIG. 2. T/(1-T) plotted vs. $\omega^{2}$ for the 7 thickest films from set 3; The solid lines are Drude Model fits, as described in the text. The inset shows the plasma frequency for these films (open symbols) and three quenched films from sets 1 and 3 (filled symbols) as a function of the dc sheet resistance, with the flat line representing the plasma frequency of bulk lead obtained from Ref. 8 . 
Fig. 1

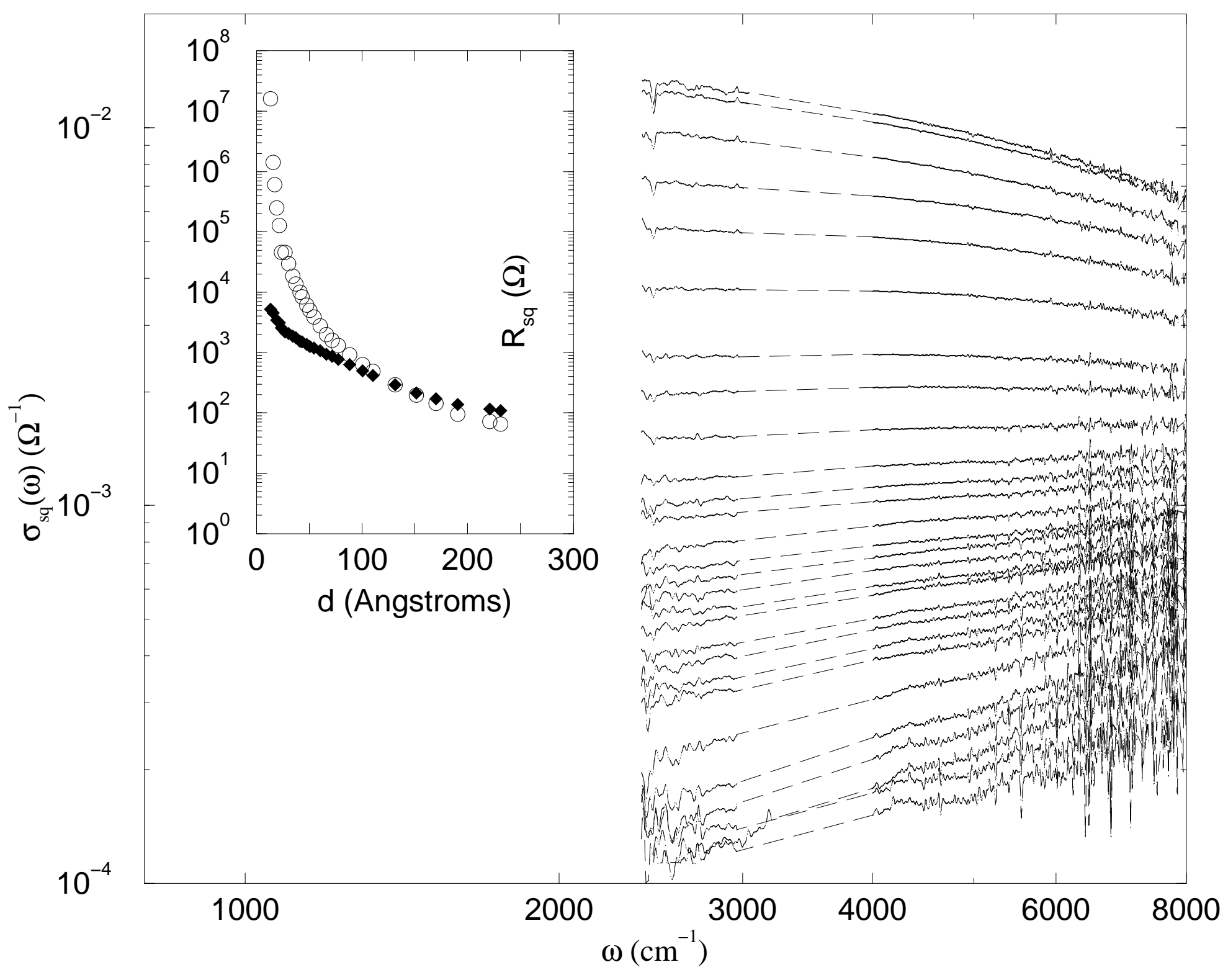


Fig. 2

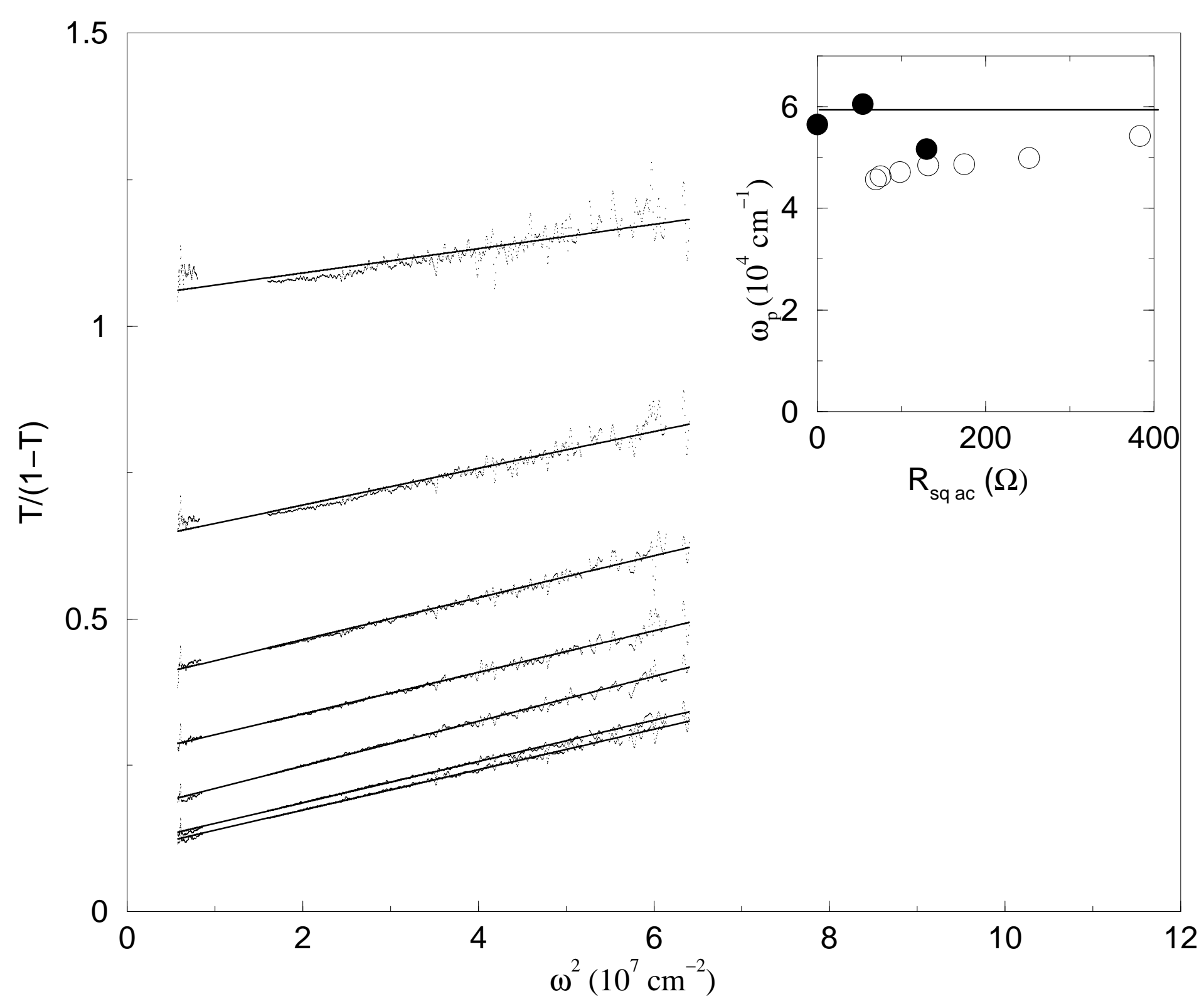




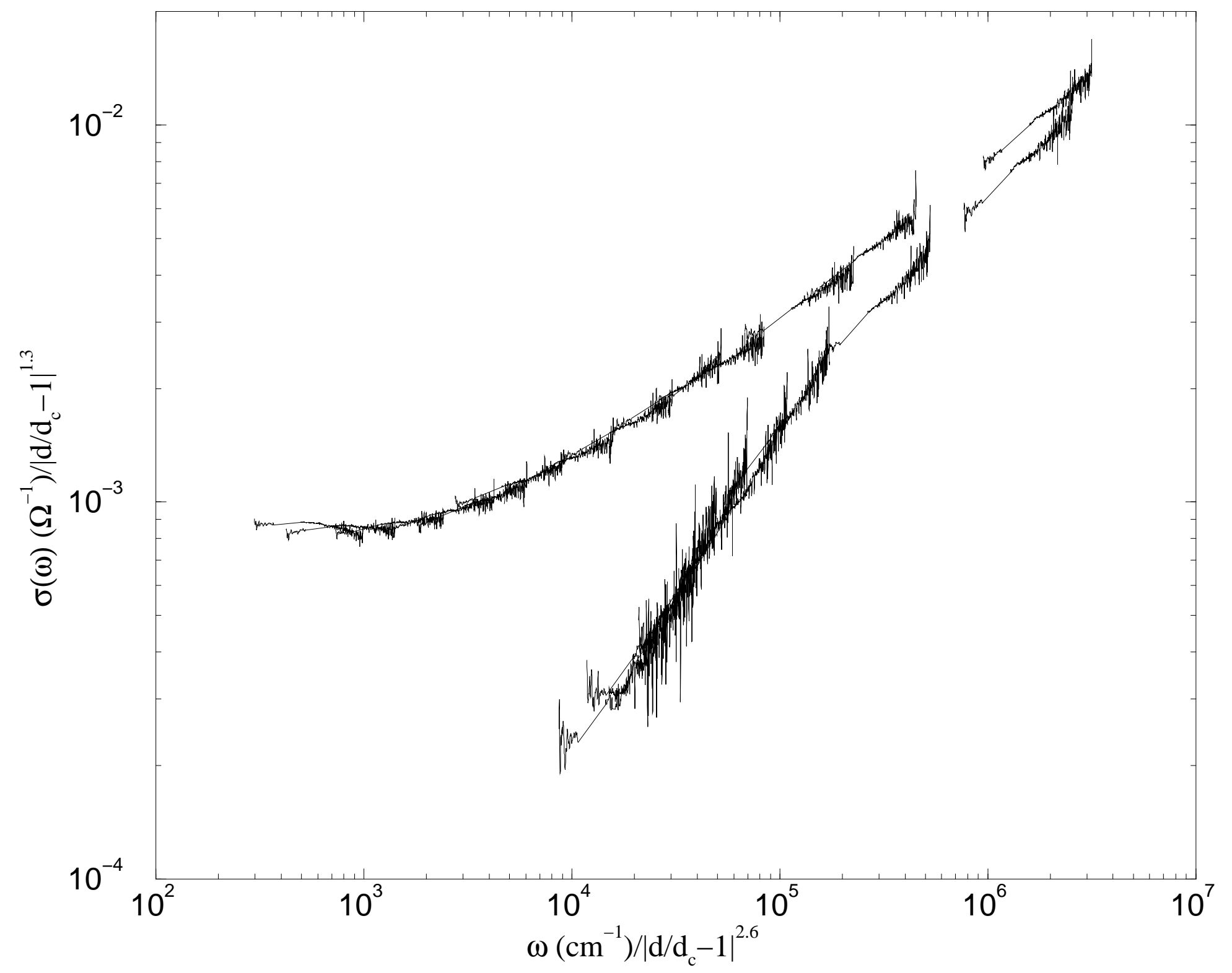

